\title{
Optimal Sensor Placement for Underground Tunnel Monitoring via Wireless Sensor Networks
}

\author{
Yonggang $\mathrm{Li} \mathbb{D}^{\mathrm{C}}{ }^{1} \mathrm{Bin}_{\mathrm{He}}{ }^{2}$ and Youming Wang ${ }^{1}$ \\ ${ }^{1}$ Department of automation, Xi'an University of Posts and Telecommunications, Xi'an, 710121, China \\ ${ }^{2}$ Electrical Engineering Department, Tongji University, Shanghai 200092, China \\ Correspondence should be addressed to Yonggang Li; activebee1979@163.com
}

Received 15 October 2020; Revised 22 November 2020; Accepted 11 December 2020; Published 29 January 2021

Academic Editor: Jose M. Gimenez-Guzman

Copyright ( 2021 Yonggang Li et al. This is an open access article distributed under the Creative Commons Attribution License, which permits unrestricted use, distribution, and reproduction in any medium, provided the original work is properly cited.

\begin{abstract}
In replace of human labor, wireless sensor networks (WSNs) are increasingly being utilized to perform structural health monitoring of underground tunnel. Due to its complex environment, the deployment of sensor nodes poses a big challenge to related staff. How to use the optimal number of sensor nodes deployed in the underground tunnel to obtain a satisfactory monitoring is our main consideration. In this paper, we propose a deployment strategy based on the optimal index to provide guidelines for sensor node placement. The objective of the strategy is to put sensor nodes in a proper site to gain maximum sensing information, thus eliminating redundant sensor nodes as well as saving costs.
\end{abstract}

\section{Introduction}

Sensor node placement should meet two basic requirements: coverage and connectivity. Sensor nodes deployed in a sensing field have the ability to sense and display the full information of objects. Even if there appear many kinds of holes such as coverage holes, routing holes, jamming holes, and wormholes in a specified region of interest, related sensor nodes depend on self-healing algorithms to detect and recover these holes in order to achieve the full coverage [1-3]. Moreover, sensor nodes are required to communicate with each other, thus making the delivery of the sensing information possible. A network is said to be fully multihop connected if it has at least one wireless multihop path linking each sensor node to each other sensor node. Especially in the presence of sensor failures, a network still remains connected relying on some connectivity modes of WSNs [4-6]. Coverage and connectivity together are two fundamental metrics to evaluate the performance of WSNs $[7,8]$.

There is growing interest in deploying wireless sensors in the underground tunnel to perform structural health monitoring including damage detection, crack detection, and water leakage detection $[9,10]$. However, a complex under- ground environment brings a considerable difficulty in deploying sensor nodes. Gupta et al. reviewed the following optimization criteria for optimal placement of piezoelectric sensors and actuators on a smart structure such as maximizing modal forces/moments, maximizing degree of controllability/observability, and minimizing spill-over effects [11]. Nestorovic and Trajkov developed a general approach to optimal actuator and sensor placement applicable for beam and plate structures as well as other complex geometries of structures, which has advantage over modal truncation and mathematical criteria due to its selection of the modes of interest [12]. Following the line of this research, we expand the scenario from the beam and plate structures to the underground environment. This paper attaches importance to optimization criteria based on $H_{2}$ and $H_{\infty}$ norms, which are calculated for all possible candidate locations.

The remainder of this paper is organized as follows. Section 2 points out sensor deployment issues existing in placement process. Section 3 is concerned with the state space of the underground tunnel. The optimal placement index is derived based on the balanced model reduction of state space. In Section 4, the placement case of the underground tunnel based on the optimal placement index is proposed to gain 
insight into sensor node deployment. Finally, the paper is concluded in Section 5.

\section{Sensor Node Deployment Issue}

A disk model is adopted that a sensor has a fixed sensing radius, and sensing area is characterized by a regular disk [13]. Furthermore, it is assumed that everything with this area can be perfectly observed, and everything outside cannot be measured by the sensors. If sensor nodes are densely deployed in sensor field, sensing areas of sensor nodes are overlapped, thus producing redundant reformation in WSNs (see Figure 1(a)). On the other hand, if sensor nodes are sparsely deployed in the sensor field, sensing areas of sensor nodes are independent of each other, thus causing unsatisfactory coverage (see Figure 1(c)). The purpose of optimal sensor node is to achieve the maximum sensing information using the minimum number of sensors deployed in sensor field.

Let $S$ denotes the sensor area of a sensor, subscripts $i$ and $j$ represent different sensors, and $r$ the radius of sensor area, $S\left(x_{i}, y_{j}\right)$ be spatial coordinates of sensor $i$. The three relationships of sensor node deployment are expressed as follows:

$$
\left\{\begin{array}{l}
S_{i} \cap S_{j}=S_{c}, \text { if } \sqrt{\left(x_{i}-x_{j}\right)^{2}+\left(y_{i}-y_{j}\right)^{2}}<2 r \\
S_{i} \cap S_{j}=c, \text { if } \sqrt{\left(x_{i}-x_{j}\right)^{2}+\left(y_{i}-y_{j}\right)^{2}}=2 r \\
S_{i} \cap S_{j}=\varphi, \text { if } \sqrt{\left(x_{i}-x_{j}\right)^{2}+\left(y_{i}-y_{j}\right)^{2}}>2 r
\end{array}\right.
$$

where $S_{c}$ represents the overlapping region between two sensor sensing areas, $c$ represents the point of contact. Let the whole area of an event area be $Q$, and the number of sensors $n$ should satisfy the following inequality:

$$
n>\frac{Q}{S}
$$

\section{Theoretical Derivation}

3.1. State Space of the Underground Tunnel. The equation of motion of the underground tunnel can be approximately expressed as follows:

$$
M \ddot{q}+D_{d} \dot{q}+K q=F,
$$

where $M$ represents the mass matrix, $D_{d}$ the damping matrix, and $K$ the stiffness matrix. The vector $q$ contains all node-wise control forces $F_{C}$ :

$$
q^{T}=\left[\begin{array}{lllll}
u_{1}^{T} \phi_{1} u_{2}^{T} & \phi_{2} & \cdots & u_{n}^{T} & \phi_{n}
\end{array}\right]
$$

The total load vector $F$ is split for the purpose of the control design into the vector of external forces $F_{E}$ and the vector of the control forces $F_{C}$ :

$$
F=F_{E}+F_{C}=\bar{E} \bar{f}(t)+\bar{B} \bar{u}(t)=B_{0} u,
$$

where matrix $B_{0}$ represents the input matrix, and vector $u$ includes all model inputs. For the controller design purposes, Eq. (3) is accompanied by the output equation in the form

$$
y=C_{0 q} q+C_{0 v} \dot{q}
$$

where in general case $C_{0 q}$ represents the output displacement matrix, $C_{0 v}$ represents the output velocity matrix, and $q$ is the generalized displacement vector containing all degrees of freedom. Solution of Eq. (3) is determined in the form $q=\varphi e^{j \omega t}$ by solving the eigenvalue problem for a homogeneous case

$$
\operatorname{det}\left(K-\omega^{2} M\right)=0 \text {, }
$$

where $\omega_{i}$ is the $i$ th natural frequency and $\varphi_{i}$ is the $i$ th mode shape vector. The solution can be represented in the matrix form by the matrix of natural eigenfrequencies $\Omega$ and the modal matrix $\Phi$.

$$
\begin{gathered}
\Omega=\left[\begin{array}{cccc}
\omega_{1} & 0 & \cdots & 0 \\
0 & \omega_{2} & \cdots & 0 \\
\cdots & \cdots & \vdots & \cdots \\
0 & 0 & \cdots & \omega_{n}
\end{array}\right], \\
\Phi=\left[\begin{array}{cccc}
\varphi_{11} & \varphi_{12} & \cdots & \varphi_{n 1} \\
\varphi_{12} & \varphi_{22} & \cdots & \varphi_{n 2} \\
\cdots & \cdots & \vdots & \cdots \\
\varphi_{1 n_{d o f}} & \varphi_{1 n_{d o f}} & \cdots & \varphi_{1 n_{d o f}}
\end{array}\right]=\left[\begin{array}{llll}
\varphi_{1} & \varphi_{2} & \cdots & \varphi_{n}
\end{array}\right] .
\end{gathered}
$$

The nodal model representation (3) is transformed into a model in modal coordinates applying the following modal transformation:

$$
q=\Phi q_{m}
$$

where $q_{m}$ represents the vector of modal degrees of freedom or generalized modal displacements. Since the mass and the stiffness matrix are symmetric and positive definite, it can be shown that the mode shapes corresponding to distinct natural frequencies are orthogonal with respect to mass and stiffness matrix

$$
\Phi^{T} M \Phi=M_{m}=\operatorname{diag}\left(m_{i}\right), \Phi^{T} K \Phi=K_{m}=\operatorname{diag}\left(m_{i} \omega_{i}^{2}\right) .
$$

After appropriate transformations taking into account the orthogonality [13], the modal model is obtained in the form of a system of decoupled equations under the assumption of proportional damping in (4)

$$
D_{d}=\alpha M+\beta K \text {. }
$$




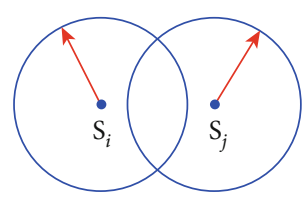

(a) Sensing area overlap

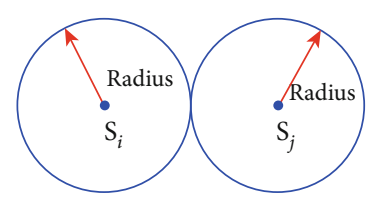

(b) Sensing area contact

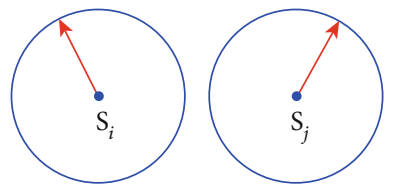

(c) Sensing area separation

Figure 1: Sensor node deployment.

By appropriate selection of the state-space vector, the state-space models of different forms can be obtained. With the coordinate transformation in the state-space form

$$
\begin{gathered}
x=\left[\begin{array}{c}
\Omega q_{m} \\
\dot{q}_{m}
\end{array}\right], \\
\left\{\begin{array}{l}
\dot{x}=A x+B u \\
y=C x+D u
\end{array} .\right.
\end{gathered}
$$

Coefficient matrices are expressed as follows:

$$
A=\left[\begin{array}{cc}
0 & \Omega \\
-\Omega & -2 z \Omega
\end{array}\right], B=\left[\begin{array}{c}
0 \\
B_{m}
\end{array}\right], C=\left[\begin{array}{ll}
C_{m q} & C_{m v}
\end{array}\right], D=0,
$$

where $\Omega^{2}=M_{m}^{-1} K_{m}, Z=\operatorname{diag}\left(\zeta_{i}\right)$ with $\zeta_{i}$ being the damping ratio of the $i$ th mode, $B_{m}=\Phi^{T} B_{0}, C_{m q}=C_{0 q} \Phi$, and $C_{m v}=C_{0 v} \Phi$.

Considering that flexible structures can be described in terms of independent coordinates, the modal state-space model can be expressed in terms of state-space realizations $\left(A_{m i}, B_{m i}, C_{m i}\right)$ for each mode $i$, with the coordinate transformation corresponding to (12)

$$
x_{i}=\left[\begin{array}{c}
\omega_{i} \dot{q}_{m} \\
q_{m}
\end{array}\right]
$$

Corresponding matrices in the realization $\left(A_{m i}, B_{m i}, C_{m i}\right)$ are determined

$$
A_{m i}=\left[\begin{array}{cc}
0 & \omega_{i} \\
-\omega_{i} & -2 \xi_{i} \omega_{i}
\end{array}\right], B_{m i}=\left[\begin{array}{c}
0 \\
b_{m i}
\end{array}\right], C_{m i}=\left[\begin{array}{cc}
\frac{C_{m q_{i}}}{\omega_{i}} & C_{m v_{i}}
\end{array}\right] .
$$

The elements of the realization $\left(A_{m i}, B_{m i}, C_{m i}\right)$ are used for assessing the optimal sensor locations based on candidate input/output transfer functions relating corresponding sensor.

3.2. Balanced Model Reduction. It is well known that the general modeling problem involves a tradeoff between complexity and accuracy of models. Simple and accurate models have the advantage over complex and inaccurate models in that the former has more rich descriptive and predictive power than the latter. So how to obtain a simple model with the same properties as the original model is the core work of model reduction.

Model reduction of large-scale dynamical systems has received a lot of attention during the last two decades. It is a crucial tool in reducing the computational complexity while preserving the properties of the original systems. It has been widely applied in many applications such as VLSI Circuits and weather forecasting.

Model reduction for linear systems is well developed based on several years of research work and intrinsic properties of linear systems. While extended to nonlinear systems, some considerations must be confronted. Thus, model reduction for nonlinear systems is much more difficult than that for linear systems. The difficulty involved in the model reduction for nonlinear systems comes from the following two facts. One is that nonlinear systems exhibit more complex behaviors than linear systems in the sense that the reduced models for nonlinear systems need to preserve much nonlinear properties. The other is that it is hard to obtain a general and universal inputoutput representation similar to the transfer function to describe nonlinear systems. Lack of representation adds the corresponding burden to the nonlinear system model reduction work.

Model reduction via balancing is gaining popularity because of its simple implement and clear presentation. Balancing is a well-known subject which dated back to a paper of Moor with the aim of using it to cope with model reduction [14]. If a linear system is in balanced form, the Hankel singular value acts as a tool to measure the influence of the corresponding state component on the output and input energy. If 
a Hankel singular value is relatively small, the impact of the corresponding state component on the output and input energy is small. Some state components corresponding to relatively large Hankel singular value are regained, and some others corresponding to relatively small Hankel singular value are eliminated, thus forming a reduced-order model. Here, a linear time-invariant system is illustrated to be familiar with the balancing model reduction.

For a linear continuous invariant system, the state-space form is given by Eq. (17).

$$
\left\{\begin{array}{l}
\dot{x}=A x+B u \\
y=C x
\end{array}\right.
$$

Reachability gramian and observability gramian which correspond to the above system are given by Eq. (4), respectively. If the system is stable and controllable, then the controllability gramian has full rank. Likewise, if the system is observable, then the observability gramian has full rank.

$$
\left\{\begin{array}{l}
P=\int_{0}^{\infty} e^{A t} B B^{T} e^{A t} d t \\
Q=\int_{0}^{\infty} e^{A^{T} t} C^{T} C e^{A t} d t
\end{array} .\right.
$$

The reachability gramian and observability gramian satisfy the following Lyapunov equation given by (19).

$$
\left\{\begin{array}{l}
A P+P A^{T}+B B^{T}=0 \\
Q A+A^{T} Q+C^{T} C=0
\end{array} .\right.
$$

The balancing truncation as one of the methods for linear system model reduction gains popularity due to the property of easy use. Its aim is to find a transformation which makes reachability gramian and observability gramian diagonal and equal.

$$
T P T^{T}=T Q T^{-1}=\left[\begin{array}{ccccc}
\sigma_{1} & & & & \\
& \sigma_{2} & & & \\
& & \ddots & & \\
& & & \sigma_{n-1} & \\
& & & & \sigma_{n}
\end{array}\right] \text {, }
$$

where is $\sigma$ Hankel singular value and $T$ is a transformation matrix. The Hankel singular values are ordered in magnitude in the diagonal line. Thus, the system is called in a balanced form. This state-space realization is called a balanced realization. The system is balancing in the following two senses [15]:

(i) $P$ and $Q$ are in a diagonal form; and (ii) $P=Q$, which means that the relationship between the input-to-state behavior and the state-to-output behavior is balanced

From the Hankel singular value, it is clear to obtain the information that what states corresponding to the Hankel singular value contribute more or less to the system behavior than other states. So eliminate the states which have little effect on the system behavior and retain the states which influence input-output behavior at most, thus completing the work of model reduction.

It can be shown that there exists a state-space transformation

$$
\bar{x}=T x .
$$

Such that the transformed system given by

$$
\left\{\begin{array}{l}
\dot{\bar{x}}=T A T^{-1} \bar{x}+T B u=\bar{A} \bar{x}+\bar{B} u \\
\bar{y}=C T^{-1} \bar{x}=\bar{C} \bar{x}
\end{array} .\right.
$$

It is more interesting to point out that gramians act as a bridge between states and energy. The idea behind gramians is that the singular values in the controllability gramian correspond to the amount of energy applied to systems in order to steer the states to the expected place, and the singular values in the observability gramian refer to the energy generated by the corresponding states.

3.3. Optimal Placement Index. Gawronski has given the proofs for modes norm such as $\mathrm{H}_{2}$ norm of a single mode, $H_{2}$ norm of a structure, $H_{\infty}$ norm of a single mode, $H_{\infty}$ norm of a structure, and $H_{2}$ and $H_{\infty}$ hybrid norm of a single mode and a system [16]. $H_{2}$ and $H_{\infty}$ hybrid norm of a single mode is expressed as follows:

$$
\left\|G_{i}\right\|_{2, \infty} \cong \sqrt{\sum_{j=1}^{r}\left\|G_{i, j}\right\|_{(2, \infty)}^{2}} i=1, \cdots n .
$$

Index $i$ regards the $i$ th mode, and in the case of the norm for a whole structure, it should be omitted. Here, $p$ represents the number of sensors. $\mathrm{H}_{2}$ norm of the $i$ th mode with a single sensor corresponding to the $k$ th position is given by

$$
\left\|G_{i}^{k}\right\|_{2}=\frac{\left\|B_{m i}\right\|_{2}\left\|C_{m i}^{k}\right\|_{2}}{2 \sqrt{\zeta_{i} \omega_{i}}}
$$

Similarity, $\mathrm{H}_{2}$ norm of the $i$ th mode with a single sensor corresponding to the $k$ th position is given by

$$
\left\|G_{i}^{k}\right\|_{\infty}=\frac{\left\|B_{m i}\right\|_{2}\left\|C_{m i}^{k}\right\|_{2}}{2 \zeta_{i} \omega_{i}} .
$$




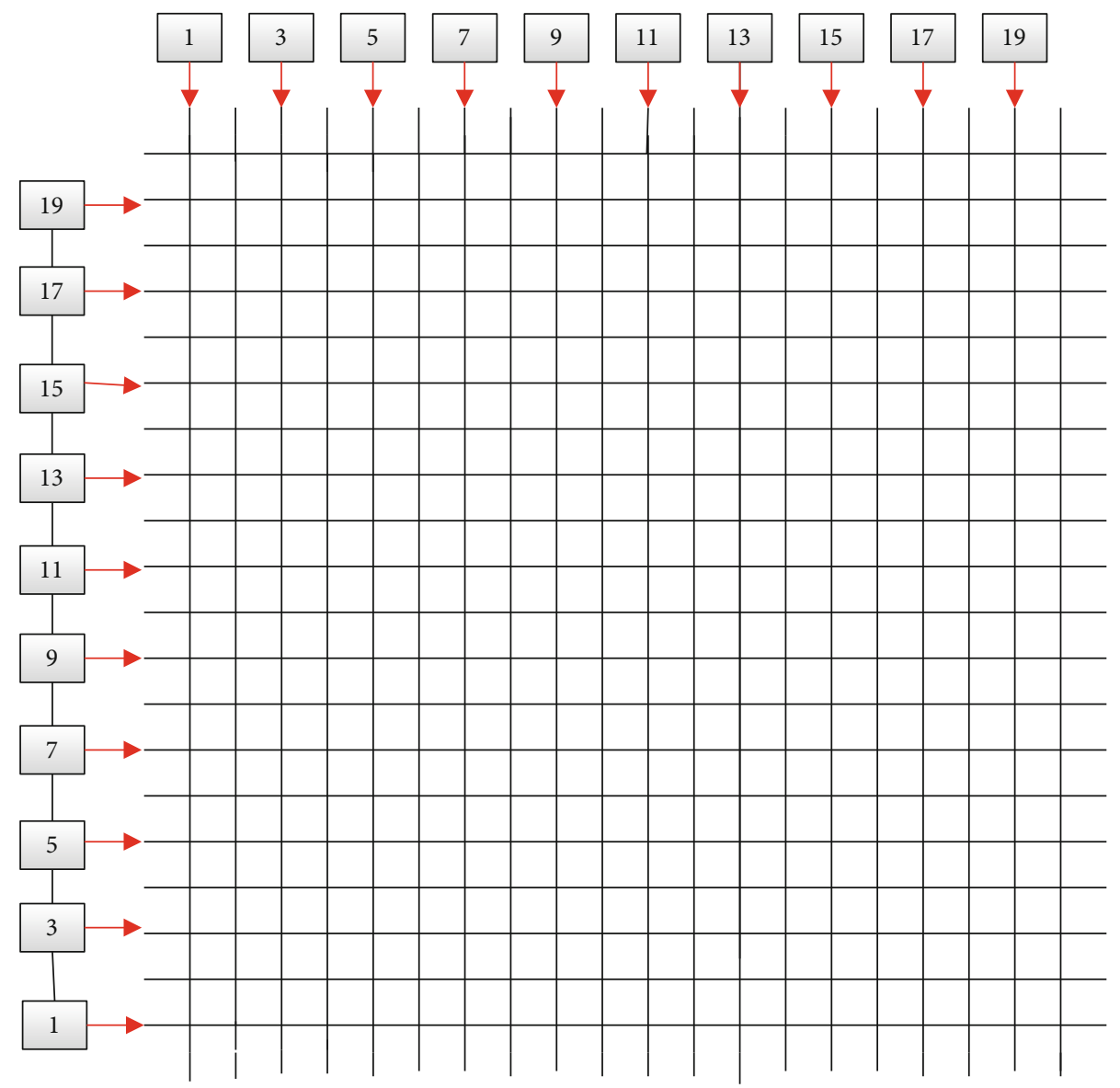

FIGURE 2: Candidate locations for sensors.

TABLE 1: Candidate locations based on the largest placement indices.

\begin{tabular}{lccc}
\hline Mode & $H_{2}$ & $H_{\infty}$ & Observability index \\
\hline 1 & $(10,10)$ & $(10,10)$ & $(10,10)$ \\
2 & $(10,5),(10,6)(10,15),(10,16)$ & $(10,5),(10,6)(10,15),(10,16)$ & $(10,5),(10,6)(10,15),(10,16)$ \\
4 & $(10,4),(10,1),(10,18)$ & $(10,4),(10,11),(10,18)$ & $(10,4),(10,11),(10,18)$ \\
5 & $(5,6),(5,16)(15,6),(15,16)$ & $(5,6),(5,16)(15,6),(15,16)$ & $(5,6),(5,16)(15,6),(15,16)$ \\
\hline
\end{tabular}

The placement index is defined based on the $\mathrm{H}_{2}$ norm and $H_{\infty}$ norm. For a clear overview, the placement indices are arranged within appropriate placement matrices

$N_{(2, \infty)}=\left[\begin{array}{cccccc}\eta_{1}^{1}(2, \infty) & \eta_{1}^{2}(2, \infty) & \cdots & \eta_{1}^{k}(2, \infty) & \cdots & \eta_{1}^{p}(2, \infty) \\ \eta_{2}^{1}(2, \infty) & \eta_{2}^{2}(2, \infty) & \cdots & \eta_{2}^{k}(2, \infty) & \cdots & \eta_{2}^{p}(2, \infty) \\ \cdots & \cdots & \vdots & \cdots & \vdots & \cdots \\ \eta_{i}^{1}(2, \infty) & \eta_{i}^{2}(2, \infty) & \cdots & \eta_{i}^{k}(2, \infty) & \cdots & \eta_{i}^{p}(2, \infty) \\ \cdots & \cdots & \vdots & \cdots & \vdots & \cdots \\ \eta_{n}^{1}(2, \infty) & \eta_{n}^{2}(2, \infty) & \cdots & \eta_{n}^{k}(2, \infty) & \cdots & \eta_{n}^{p}(2, \infty)\end{array}\right]$,

where each row corresponds to the $i$ th mode and each column to the $k$ th sensor. For the objective function in terms of the $\mathrm{H}_{2}$ norm, sensor (subscript $s$ ) placement indices are determined as the root mean square sum of the column-wise elements

$$
\eta_{s}^{k}=\sqrt{\sum_{i=1}^{n}\left(\eta_{i}^{k}\right)^{2}}, k=1, \cdots, r
$$

For the objective function in terms of the $H_{\infty}$ norm, the sensor placement index is the largest index over all modes

$$
\eta_{s}^{k}=\max _{i}\left(\eta_{i}^{k}\right), i=1, \cdots, n, k=1, \cdots, r
$$




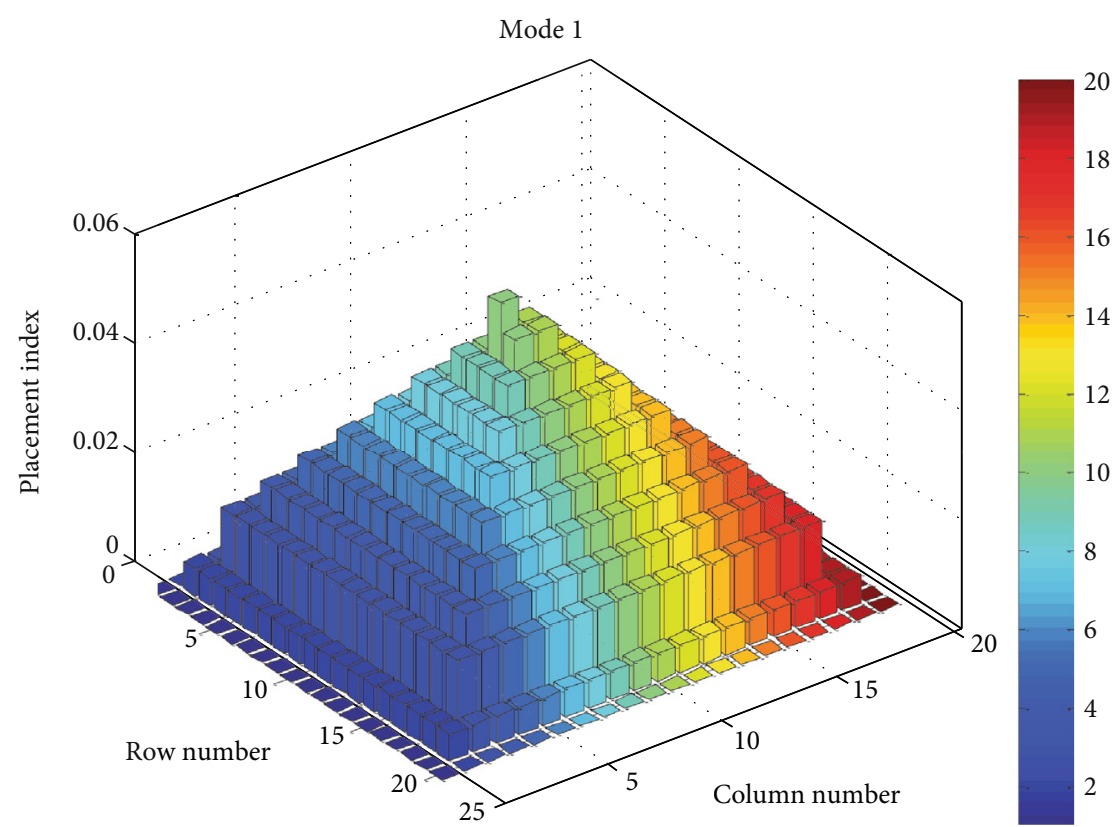

(a) Placement indices for mode 1

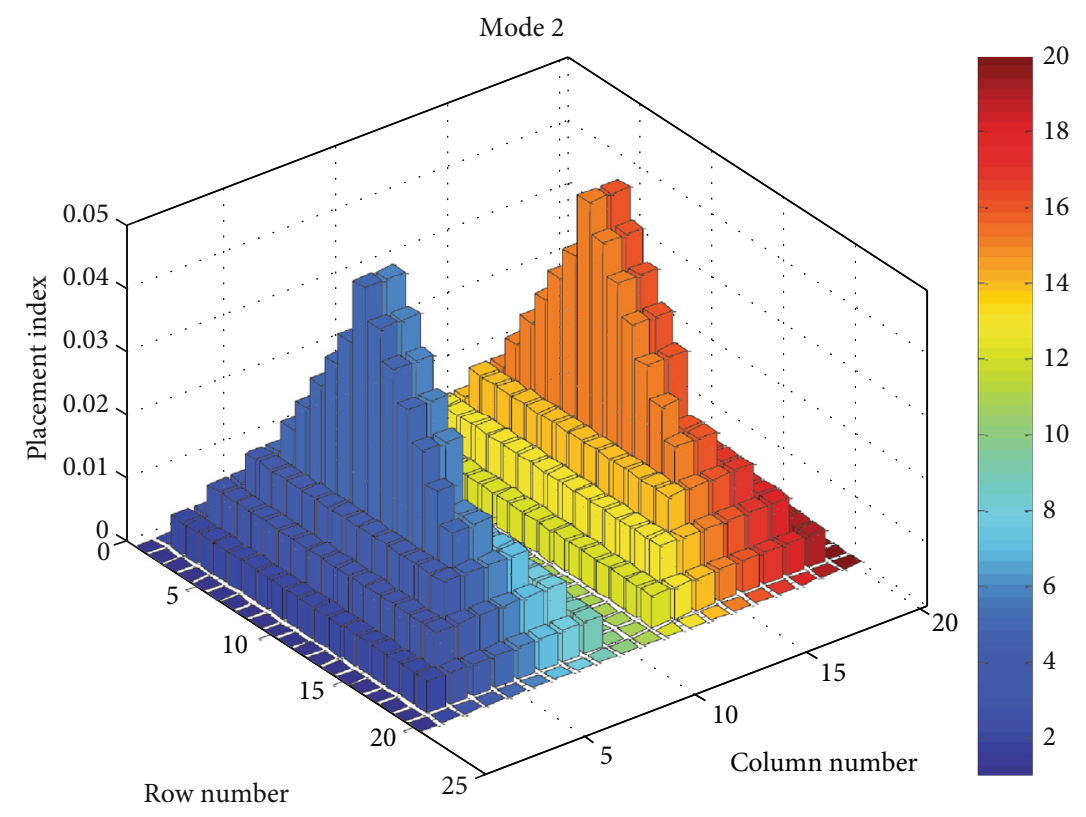

(b) Placement indices for mode 2

FIgURE 3: Continued. 


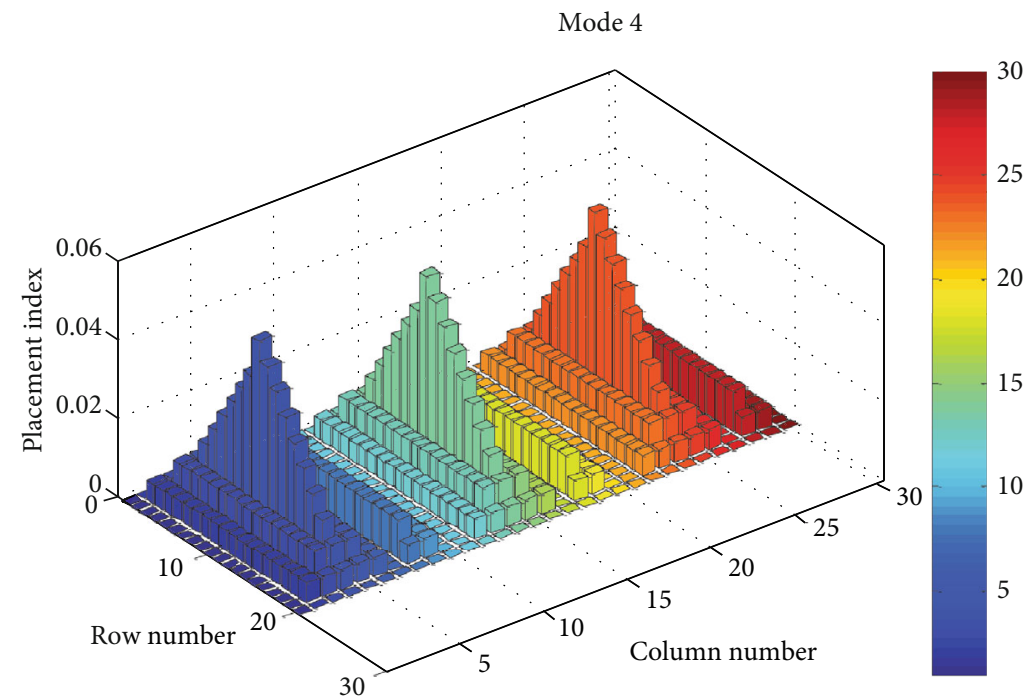

(c) Placement indices for mode 4

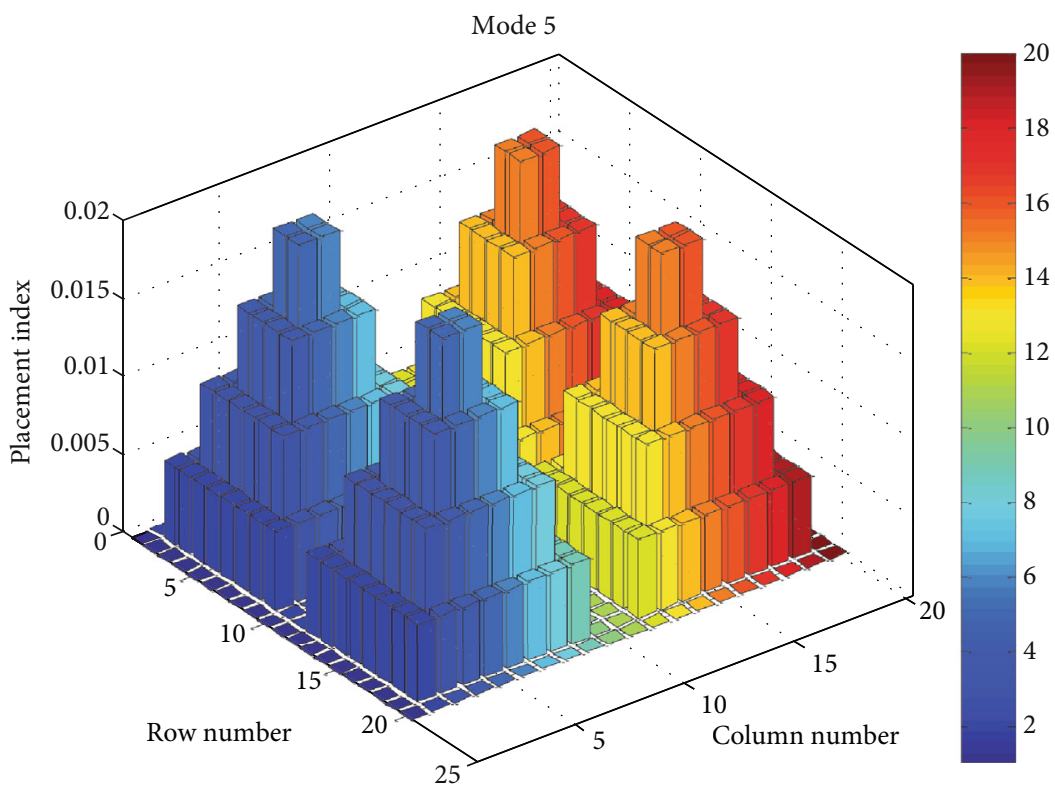

(d) Placement indices for mode 5

Figure 3: Placement indices for the underground tunnel.

In order to draw comparison with the placement indices above, we introduce observability indices for the $k$ th sensor location given by

$$
v_{i}(k)=\frac{\varphi_{i}^{T} C_{k}^{T} C_{k} \varphi_{i}}{\varphi_{i}^{T} \varphi_{i}}
$$

The placement indices including $\mathrm{H}_{2}$ norm placement indices, $H_{\infty}$ norm placement indices, and observability indices provide guidelines for sensor deployment in the underground tunnel.

\section{Optimal Sensor Placement in the Underground Tunnel}

4.1. Mesh Generation of the Underground Tunnel. Shanghai underground tunnel belongs to shield tunnel. The sensor nodes are distributed on the ring segments with $20 \mathrm{~m}$ away between each two cross sections. There exists at least one routing node located on the cross section. The sink node is located outside the underground tunnel with the minimal average distance to each routing node, thus saving the energy consumption during data transportation process. According to the deployment principles, the grid cell of the underground tunnel cross section is obtained using mesh generation. Each grid cell represents the candidate location for the 
sensor. The placement indices affect the deployment results. The meshing of the underground tunnel cross section is represented in Figure 2. Here, the corresponding rows and columns are numerated for a better preview.

4.2. Sensor Deployment in the Underground Tunnel. Each grid cell has its specific two-dimensional coordinate expressed by the row and the column shown in Figure 2. The placement indices of all grid cells are computed, and the corresponding largest values are selected to construct the table for candidate locations shown in Table 1. The simulation results of mode 3 are the same as those of mode 2 . So the corresponding results of mode 3 are removed from Table 1.

It can be seen that qualitative representations of the placement indices based on $H_{2}$ and $H_{\infty}$ hybrid norm as well as the observability index. The $3 \mathrm{D}$ bar diagrams of the placement indices based on the observability index are shown in Figure 3.

Each maximum placement index has the highest peak as shown in the 3D bar diagram. The grid cell corresponding to the highest peak is the optimal location for a sensor deployment. The optimal locations for sensor deployment are not the same under different modes. Moreover, the placement location has no relationship with mode order. It was found out that the placement condition is dominated by low order modes. Our research is limited to the first five modes.

It is noted that the probability that a node will be deployed in a candidate location where the placement index is equal to 1 , regardless of nodes specific distributions such as Poisson distribution and exponential distribution.

\section{Conclusion}

In this paper, the optimization approaches for sensor node placement for the underground tunnel are proposed, based on balanced reduction of space state models. Based on $\mathrm{H}_{2}$ and $H_{\infty}$ norms, the optimal placement indices are obtained to determine optimal positions from many candidate locations. The pursuit of optimal position is crucial for sensor nodes to obtain maximal sensing information, thereby performing a good monitoring of the underground tunnel.

\section{Data Availability}

There is no data availability statement.

\section{Conflicts of Interest}

The authors declare that they have no conflicts of interest.

\section{Acknowledgments}

This work was supported by the National Natural Science Foundation of China (Grant No. 51875457).

\section{References}

[1] M. R. Senouci, A. Mellouk, and K. Assnoune, "Localized movement-assisted sensor deployment algorithm for hole detection and healing," IEEE Transactions on Parallel and Distributed Systems, vol. 25, no. 5, pp. 1267-1277, 2014.
[2] A. Nadeem, S. K. Salil, and J. Sanjay, "A pragmatic approach to area coverage in hybrid wireless sensor networks," wireless communication and mobile computing, vol. 11, no. 1, pp. 2345, 2011.

[3] C. Y. Chang, L. L. Huang, S. W. Chang, and Y. C. Chen, "Decentralized and energy-balanced algorithms for maintaining temporal full-coverage in full-coverage in moblile WSNs," wireless communication and mobile computing, vol. 12, no. 5, pp. 445-462, 2012.

[4] L. A. Laranjeira and G. N. Rodrigues, "Border effect analysis for reliability assurance and continuous connectivity of wireless sensor networks in presence of sensor failures," IEEE Transactions on Wireless Communications, vol. 13, no. 8, pp. 4232-4246, 2014.

[5] K. Kucuk and A. Kavak, "Connectivity analysis for wireless sensor networks with antenna array integrated central node," wireless personal communications, vol. 72, pp. 1361-1371, 2013.

[6] S. B. He, J. M. Chen, and Y. X. Sun, "Coverage and connectivity in duty-cycled wireless sensor networks for event monitoring," IEEE Transactions on Parallel and Distributed Systems, vol. 23, no. 3, pp. 475-482, 2012.

[7] C. Zhu, C. L. Zheng, L. Shu, and G. J. Han, "A survey on coverage and connectivity issues in wireless sensor networks," Journal of Network and Computer Applications, vol. 35, no. 2, pp. 619-632, 2012.

[8] A. Chosh and S. K. Das, "Coverage and connectivity issues in wireless sensor networks: a survey," Pervasive and Mobile Computing, vol. 4, pp. 303-334, 2008.

[9] A. Hada, K. Soga, R. Liu, and I. J. Wassell, "Lagrangian heuristic method for the wireless sensor network design problem in railway structural health monitoring," Mechanical System and Signal Processing, vol. 28, pp. 20-35, 2012.

[10] C. Hirai and K. Soge, “An experimental model of relay development planning tool for wireless sensor network system to monitor civil engineering structure," Proceeding of the 19th LASTED International Conference Parallel and Distributed Computing and Network (PDCN 2010), 2010, pp. 164-171, Innsbruck, Austria, 2010.

[11] V. Gupta, M. Sharma, and N. Thakur, "Optimization criteria for optimal placement of piezoelectric sensors and actuators on a smart structure: a technical review," Journal of Intelligent Material Systems and Structures, vol. 21, no. 12, pp. 12271243, 2010.

[12] T. Nestorovic and M. Trajkov, "Optimal actuator and sensor placement based on balanced reduced models," Mechanical Systems and Signal Processing, vol. 36, no. 2, pp. 271-289, 2013.

[13] S. Meguerdichian, K. Koushanfar, G. Qu, G. Veltri, and M. Potkonjak, "Exposure in wireless sensor networks: theory and practical solutions," Wireless Networks, vol. 8, no. 5, pp. 443-454, 2002.

[14] B. C. Moore, "Principal component analysis in linear systems: controllability, observability, and model reduction," IEEE Transaction on Automatic Control, vol. 26, no. 1, pp. 17-32, 1981.

[15] K. Fujimoto and J. M. A. Scherpen, "Balanced realization and model order reduction for nonlinear systems based on singular value analysis," SIAM Journal on Control and Optimization, vol. 48, no. 7, pp. 4591-4623, 2010.

[16] W. Gawronski, Advanced Structural Dynamics and Active Control of Structures, Springer, New York, 2004. 